\title{
Divisor class groups of graded hypersurfaces
}

\author{
Anurag K. Singh and Sandra Spiroff
}

\begin{abstract}
We demonstrate how some classical computations of divisor class groups can be obtained using the theory of rational coefficient Weil divisors and related results of Watanabe.
\end{abstract}

\section{Introduction}

The purpose of this note is to provide a simple technique to compute divisor class groups of affine normal hypersurfaces of the form

$$
k\left[z, x_{1}, \ldots, x_{d}\right] /\left(z^{n}-g\right),
$$

where $g$ is a weighted homogeneous polynomial in $x_{1}, \ldots, x_{d}$ of degree relatively prime to $n$. We use the theory of rational coefficient Weil divisors due to Demazure [3] and related results of Watanabe [14. This provides an alternative approach to various classical examples found in Samuel's influential lecture notes [10, as well as to computations due to Lang [6] and Scheja and Storch [11. While the computations we present here are subsumed by those of [11, our techniques are different. A key point in our approach is that the projective variety defined by a hypersurface as above is weighted projective space over $k$, and this makes for straightforward, elementary calculations.

Watanabe [14, page 206] pointed out that $\mathbb{Q}$-divisor techniques can be used to recover the classification of graded factorial domains of dimension two, originally due to Mori $\mathbf{8}$. Robbiano has applied similar methods to a study of factorial and almost factorial schemes in weighted projective space [9].

\section{2. $\mathbb{Q}$-divisors}

We review some material from [3] and [14]. Let $k$ be a field, and let $X$ be a normal irreducible projective variety over $k$, with rational function field $k(X)$.

A rational coefficient Weil divisor or a $\mathbb{Q}$-divisor on $X$ is a $\mathbb{Q}$-linear combination of irreducible subvarieties of $X$ of codimension one. Let $D=\sum n_{i} V_{i}$ be a $\mathbb{Q}$-divisor, where $V_{i}$ are distinct. Then $\lfloor D\rfloor$ is defined as

$$
\lfloor D\rfloor=\sum\left\lfloor n_{i}\right\rfloor V_{i},
$$

2000 Mathematics Subject Classification. Primary 13C20, Secondary 14C20.

The first author was supported by the NSF under grants DMS 0300600 and DMS 0600819.

(C) 0000 (copyright holder) 
where $\lfloor n\rfloor$ denotes the greatest integer less than or equal to $n$. We set

$$
\mathcal{O}_{X}(D)=\mathcal{O}_{X}(\lfloor D\rfloor) \text {. }
$$

If each coefficient $n_{i}$ occurring in $D$ is nonnegative, we say that $D \geqslant 0$.

A $\mathbb{Q}$-divisor $D$ is ample if $n D$ is an ample Cartier divisor for some $n \in \mathbb{N}$. In this case, the generalized section ring corresponding to $D$ is the ring

$$
R(X, D)=\oplus_{j \geqslant 0} H^{0}\left(X, \mathcal{O}_{X}(j D)\right) .
$$

If $R=R(X, D)$, then the $n$-th Veronese subring of $R=R(X, D)$ is the ring

$$
R^{(n)}=\oplus_{j \geqslant 0} H^{0}\left(X, \mathcal{O}_{X}(j n D)\right)=R(X, n D) .
$$

The following theorem, due to Demazure, implies that a normal $\mathbb{N}$-graded ring $R$ is determined by a $\mathbb{Q}$-divisor on $\operatorname{Proj} R$.

Theorem 2.1. 3, 3.5]. Let $R$ be an $\mathbb{N}$-graded normal domain, finitely generated over a field $R_{0}$. Let $T$ be a homogeneous element of degree 1 in the fraction field of $R$. Then there exists a unique ample $\mathbb{Q}$-divisor $D$ on $X=\operatorname{Proj} R$ such that

$$
R=\oplus_{j \geqslant 0} H^{0}\left(X, \mathcal{O}_{X}(j D)\right) T^{j} .
$$

We next recall a result of Watanabe, which expresses the divisor class group of $R$ in terms of the divisor class group of $X$ and a $\mathbb{Q}$-divisor corresponding to $R$.

Theorem 2.2. 14, Theorem 1.6] Let $X$ be a normal irreducible projective variety over a field. Assume $\operatorname{dim} X \geqslant 1$ and let $D=\sum_{i=1}^{r}\left(p_{i} / q_{i}\right) V_{i}$ be a $\mathbb{Q}$-divisor on $X$ where $V_{i}$ are distinct irreducible subvarieties, $p_{i}, q_{i} \in \mathbb{Z}$ are relatively prime, and $q_{i}>0$. Set

$$
R=\oplus_{j \geqslant 0} H^{0}\left(X, \mathcal{O}_{X}(j D)\right) T^{j} .
$$

Then there is an exact sequence

$$
0 \longrightarrow \mathbb{Z} \stackrel{\theta}{\longrightarrow} \mathrm{Cl}(X) \longrightarrow \mathrm{Cl}(R) \longrightarrow \operatorname{coker} \alpha \longrightarrow 0,
$$

where $\theta(1)=\operatorname{lcm}\left(q_{i}\right) \cdot D$, and $\alpha: \mathbb{Z} \longrightarrow \oplus_{i=1}^{r} \mathbb{Z} / q_{i} \mathbb{Z}$ is the map $1 \mapsto\left(p_{i} \bmod q_{i}\right)_{i}$.

In the exact sequence above, coker $\alpha$ is always a finite group. Moreover, if $X$ is projective space, a Grassmannian variety, or a smooth complete intersection in $\mathbb{P}^{n}$ of dimension at least three, then $\operatorname{Cl}(X)=\mathbb{Z}$. It follows that, in these cases, the divisor class group of $R(X, D)$ is finite for any ample $\mathbb{Q}$-divisor $D$ on $X$, and hence that $R(X, D)$ is almost factorial in the sense of Storch $\mathbf{1 2}$.

Lipman proved that the divisor class group of a two-dimensional normal local ring $R$ with rational singularities is finite, [7, Theorem 17.4]. While this is a hard result, the analogous statement for graded rings is a straightforward application of Theorem 2.2. Indeed, let $R$ be an $\mathbb{N}$-graded normal ring of dimension two, finitely generated over an algebraically closed field $R_{0}$, such that $R$ has rational singularities. Then $R$ has a negative $a$-invariant by [14, Theorem 3.3], so $H^{1}\left(X, \mathcal{O}_{X}\right)=0$ where $X=\operatorname{Proj} R$. But then $X$ is a curve of genus 0 so it must be $\mathbb{P}^{1}$, and it follows that the divisor class group of $R$ is finite.

Remark 2.3. We note some aspects of Watanabe's proof of Theorem 2.2, Let $\operatorname{Div}(X)$ be the group of Weil divisors on $X$, and let

$$
\operatorname{Div}(X, \mathbb{Q})=\operatorname{Div}(X) \otimes_{\mathbb{Z}} \mathbb{Q}
$$


be the group of $\mathbb{Q}$-divisors. For $D$ as in Theorem 2.2. set $\operatorname{Div}(X, D)$ to be the subgroup of $\operatorname{Div}(X, \mathbb{Q})$ generated by $\operatorname{Div}(X)$ and the divisors

$$
\frac{1}{q_{1}} V_{1}, \ldots, \frac{1}{q_{r}} V_{r} .
$$

Each element $E \in \operatorname{Div}(X, D)$ gives a divisorial ideal

$$
\oplus_{j \geqslant 0} H^{0}\left(X, \mathcal{O}_{X}(E+j D)\right) T^{j}
$$

of $R$, and hence an element of $\mathrm{Cl}(R)$. The map $\operatorname{Div}(X, D) \longrightarrow \mathrm{Cl}(R)$ induces a surjective homomorphism

$$
\operatorname{Div}(X, D) / \operatorname{Div}(X) \longrightarrow \mathrm{Cl}(R) / \text { image }(\mathrm{Cl}(X)) .
$$

\section{Computing divisor class groups}

The divisor class groups of affine surfaces of characteristic $p$ defined by equations of the form $z^{p^{n}}=g(x, y)$ have been studied in considerable detail; such surfaces are sometimes called Zariski surfaces. In [6 Lang computed the divisor class group of hypersurfaces of the form $z^{p^{n}}=g\left(x_{1}, \ldots, x_{d}\right)$ where $g$ is a homogeneous polynomial of degree relatively prime to $p$. The proposition below recovers 6. Proposition 3.11].

Let $A=k\left[x_{1}, \ldots, x_{d}\right]$ be a polynomial ring over a field. We say $g \in A$ is a weighted homogeneous polynomial if there exists an $\mathbb{N}$-grading on $A$, with $A_{0}=k$, for which $g$ is a homogeneous element.

Proposition 3.1. Let $R=k\left[z, x_{1}, \ldots, x_{d}\right] /\left(z^{n}-g\right)$ be a normal hypersurface over a field $k$, where $g \in k\left[x_{1}, \ldots, x_{d}\right]$ is a weighted homogeneous polynomial with degree relatively prime to $n$. Let $g=h_{1} \cdots h_{r}$, where $h_{i} \in k\left[x_{1}, \ldots, x_{d}\right]$ are irreducible polynomials. Then

$$
\mathrm{Cl}(R)=(\mathbb{Z} / n \mathbb{Z})^{r-1},
$$

and the images of $\left(z, h_{1}\right), \ldots,\left(z, h_{r-1}\right)$ form a minimal generating set for $\mathrm{Cl}(R)$.

Note that if $n \geqslant 2$, then the hypothesis that $R$ is normal forces $h_{1}, \ldots, h_{r}$ to be pairwise coprime irreducible polynomials.

Proof of Proposition 3.1. The polynomial ring $k\left[x_{1}, \ldots, x_{d}\right]$ has a grading under which $\operatorname{deg} x_{i}=c_{i}$ for $c_{i} \in \mathbb{N}$, and the degree of $g$ is an integer $m$ relatively prime to $n$. We assume, without any loss of generality, that $\operatorname{gcd}\left(c_{1}, \ldots, c_{d}\right)=1$. Consider the $\mathbb{N}$-grading on $R$ where $\operatorname{deg} x_{i}=n c_{i}$ and $\operatorname{deg} z=m$. Note that under this grading $\operatorname{deg} g=\sum \operatorname{deg} h_{i}=m n$. The $n$-th Veronese subring of $R$ is

$$
R^{(n)}=k\left[z^{n}, x_{1}, \ldots, x_{d}\right] /\left(z^{n}-g\right)=k\left[x_{1}, \ldots, x_{d}\right],
$$

which is a polynomial ring in $x_{1}, \ldots, x_{d}$. Let $X=\operatorname{Proj} R^{(n)}=\operatorname{Proj} R$.

There exist integers $s_{i}, a$, and $b$ such that $\sum_{i=1}^{d} s_{i} c_{i}=1$ and $a m+b n=1$. Consider the $\mathbb{Q}$-divisor on $X$ given by

$$
D=b \operatorname{div}(\mathbf{x})+\frac{a}{n} \operatorname{div}(g)=b \sum_{i=1}^{d} s_{i} V\left(x_{i}\right)+\frac{a}{n} \sum_{i=1}^{r} V\left(h_{i}\right),
$$

where $\mathbf{x}=x_{1}^{s_{1}} \cdots x_{d}^{s_{d}}$. We claim that

$$
R=\oplus_{j \geqslant 0} H^{0}\left(X, \mathcal{O}_{X}(j D)\right) T^{j},
$$


where $T=z^{a} \mathbf{x}^{b}$ is a homogeneous degree 1 element of the fraction field of $R$. First note that $\lfloor a m / n\rfloor=\lfloor(1-b n) / n\rfloor=-b$, so

$$
\lfloor m D\rfloor=b m \operatorname{div}(\mathbf{x})+\left\lfloor\frac{a m}{n}\right\rfloor \operatorname{div}(g)=b m \operatorname{div}(\mathbf{x})-b \operatorname{div}(g) .
$$

Consequently $\operatorname{deg}\lfloor m D\rfloor=0$, and $H^{0}\left(X, \mathcal{O}_{X}(m D)\right) T^{m}$ is the $k$-vector space spanned by the element

$$
\mathbf{x}^{-b m} g^{b} T^{m}=\mathbf{x}^{-b m}\left(z^{n}\right)^{b}\left(z^{a} \mathbf{x}^{b}\right)^{m}=z^{b n+a m}=z .
$$

Let $c=c_{t}$ for an integer $1 \leqslant t \leqslant d$. Then $n c D=b n c \operatorname{div}(\mathbf{x})+a c \operatorname{div}(g)$ has degree $n c$, and $H^{0}\left(X, \mathcal{O}_{X}(n c D)\right) T^{n c}$ contains the element

$$
x_{t} \mathbf{x}^{-b n c} g^{-a c} T^{n c}=x_{t} \mathbf{x}^{-b n c}\left(z^{n}\right)^{-a c}\left(z^{a} \mathbf{x}^{b}\right)^{n c}=x_{t} .
$$

To prove the claim (3.1.1), it remains to verify that $z, x_{1}, \ldots, x_{d}$ are $k$-algebra generators for the ring $\oplus_{j \geqslant 0} H^{0}\left(X, \mathcal{O}_{X}(j D)\right) T^{j}$. An arbitrary positive integer $j$ can be written as $u m+v n$ for $0 \leqslant u \leqslant n-1$. We then have

$$
\begin{aligned}
\lfloor j D\rfloor & =b(u m+v n) \operatorname{div}(\mathbf{x})+\left\lfloor\frac{a(u m+v n)}{n}\right\rfloor \operatorname{div}(g) \\
& =b(u m+v n) \operatorname{div}(\mathbf{x})+(v a-u b) \operatorname{div}(g),
\end{aligned}
$$

which has degree $v n$. Consequently $H^{0}\left(X, \mathcal{O}_{X}(j D)\right) T^{j}$ vanishes if $v$ is negative, and for nonnegative $v$, it is spanned by elements

$$
\mu \mathbf{x}^{-b(u m+v n)} g^{-v a+u b} T^{u m+v n}=\mu z^{u},
$$

for monomials $\mu$ in $x_{i}$ of degree $v$. This completes the proof of (3.1.1).

Since $n D$ has integer coefficients, the exact sequence of Theorem 2.2 for the divisor $n D$ and corresponding ring $R^{(n)}$ reduces to

$$
0 \longrightarrow \mathbb{Z} \stackrel{\theta}{\longrightarrow} \mathrm{Cl}(X) \longrightarrow \mathrm{Cl}\left(R^{(n)}\right) \longrightarrow 0,
$$

where $\theta(1)=n D$. Since $R^{(n)}$ is a polynomial ring, and hence factorial, it follows that $n D$ generates $\mathrm{Cl}(X)$. Next, consider the exact sequence applied to the divisor $D$ and corresponding ring $R$, i.e., the sequence

$$
0 \longrightarrow \mathbb{Z} \stackrel{\theta}{\longrightarrow} \mathrm{Cl}(X) \longrightarrow \mathrm{Cl}(R) \longrightarrow \operatorname{coker} \alpha \longrightarrow 0 \text {. }
$$

The lcm of the denominators occurring in $D$ is $n$, so we once again have $\theta(1)=n D$. Consequently $\theta$ is an isomorphism and $\operatorname{Cl}(R)=\operatorname{coker} \alpha$, where

$$
\alpha: \mathbb{Z} \longrightarrow \bigoplus_{1}^{r} \mathbb{Z} / n \mathbb{Z} \quad \text { with } \quad \alpha(1)=(a, \ldots, a) .
$$

Since $a$ and $n$ are relatively prime, it follows that

$$
\mathrm{Cl}(R)=(\mathbb{Z} / n \mathbb{Z})^{r-1} .
$$

We next determine explicit generators for $\mathrm{Cl}(R)$ by Remark 2.3. The $\mathbb{Q}$-divisors

$$
E_{t}=-\frac{1}{n} V\left(h_{t}\right) \quad \text { for } 1 \leqslant t \leqslant r
$$

give a generating set for $\operatorname{Div}(X, D) / \operatorname{Div}(X)$ which surjects onto $\operatorname{Cl}(R)$. Hence the divisorial ideals

$$
\mathfrak{p}_{t}=\oplus_{j \geqslant 0} H^{0}\left(X, \mathcal{O}_{X}\left(E_{t}+j D\right)\right) T^{j} \quad \text { where } 1 \leqslant t \leqslant d,
$$


generate $\mathrm{Cl}(R)$. The computation of $\mathfrak{p}_{t}$ is straightforward, and we give a brief sketch. First note that

$$
\begin{aligned}
\left\lfloor E_{t}+m D\right\rfloor & =b m \operatorname{div}(x)+\left\lfloor\frac{a m-1}{n}\right\rfloor V\left(h_{t}\right)+\sum_{i \neq t}\left\lfloor\frac{a m}{n}\right\rfloor V\left(h_{i}\right) \\
& =b m \operatorname{div}(\mathbf{x})-b \operatorname{div}(g),
\end{aligned}
$$

so $H^{0}\left(X, \mathcal{O}_{X}\left(E_{t}+m D\right)\right) T^{m}$ is the $k$-vector space spanned by

$$
\mathbf{x}^{-b m} g^{b} T^{m}=z .
$$

Since the degree of each $x_{i}$ is a multiple of $n$, we have deg $h_{t}=n \gamma$ for some integer $\gamma$. We next compute the component of $\mathfrak{p}_{t}$ in degree $n \gamma$. Note that

$$
\left\lfloor E_{t}+n \gamma D\right\rfloor=-V\left(h_{t}\right)+b n \gamma \operatorname{div}(\mathbf{x})+a \gamma \operatorname{div}(g),
$$

so $H^{0}\left(X, \mathcal{O}_{X}\left(E_{t}+n \gamma D\right)\right) T^{n \gamma}$ is the $k$-vector space spanned by

$$
h_{t} \mathbf{x}^{-b n \gamma} g^{-a \gamma} T^{n \gamma}=h_{t} .
$$

It is now a routine verification that $z, h_{t}$ are generators for the ideal $\mathfrak{p}_{t}$, which, we note, is a height one prime of $R$. Consequently $\mathrm{Cl}(R)$ is generated by $\mathfrak{p}_{1}, \ldots, \mathfrak{p}_{r}$. Using $\sim$ to denote linear equivalence, we have

$$
n E_{t}+n \gamma D \sim 0 \quad \text { and } \quad \sum_{i=1}^{r} E_{i}+m D \sim 0,
$$

implying that $n\left[\mathfrak{p}_{t}\right]=0$ and $\sum_{i}\left[\mathfrak{p}_{i}\right]=0$ in $\mathrm{Cl}(R)$. These correspond to the calculations with divisorial ideals,

$$
\mathfrak{p}_{t}^{(n)}=h_{t} R \quad \text { and } \quad \bigcap_{i=1}^{r} \mathfrak{p}_{i}=z R,
$$

and imply, in particular, that $\left[\mathfrak{p}_{1}\right], \ldots,\left[\mathfrak{p}_{r-1}\right]$ is a generating set for $\operatorname{Cl}(R)$.

Example 3.2. We use Proposition 3.1 to compute the divisor class group of diagonal hypersurfaces

$$
R=k\left[z, x_{1}, \ldots, x_{d}\right] /\left(z^{n}-x_{1}^{m_{1}}-\cdots-x_{d}^{m_{d}}\right)
$$

where $n$ is relatively prime to $m_{i}$ for $1 \leqslant i \leqslant d$, and $k$ is a field of characteristic zero, or of characteristic not dividing each $m_{i}$.

By the Jacobian criterion, $R$ has an isolated singularity at the homogeneous maximal ideal $\mathfrak{m}$. Hence if $d \geqslant 4$, then $R$, as well as its $\mathfrak{m}$-adic completion $\widehat{R}$, are factorial by Grothendieck's parafactoriality theorem [5]; see [2] for a simple proof of Grothendieck's theorem.

Case $d=3$. The polynomial $g=x_{1}^{m_{1}}+x_{2}^{m_{2}}+x_{3}^{m_{3}}$ is irreducible since $k\left[x_{1}, x_{2}, x_{3}\right] /(g)$ is a normal domain by the Jacobian criterion. We set $\operatorname{deg} x_{i}$ to be $m_{1} m_{2} m_{3} / m_{i}$. Then $g$ is a weighted homogeneous polynomial of degree $m_{1} m_{2} m_{3}$, which is relatively prime to $n$, so Proposition 3.1 implies that $R$ is factorial. Since $R$ satisfies the Serre conditions $\left(R_{2}\right)$ and $\left(S_{3}\right)$, the completion $\widehat{R}$ is factorial as well by [4. Korollar 1.5]. The divisor class groups of rational three-dimensional Brieskorn singularities are computed in [1, Chapter IV]; see also [13.

Case $d=2$. Let $g=x_{1}^{m_{1}}+x_{2}^{m_{2}}$. If $c=\operatorname{gcd}\left(m_{1}, m_{2}\right)$, let $m_{1}=a c$ and $m_{2}=b c$, and set $\operatorname{deg} x_{1}=b$ and $\operatorname{deg} x_{2}=a$. Let $f$ be an irreducible factor of $g$. Then $f$ is homogeneous, and hence has the form $\sum a_{i j} x_{1}^{i} x_{2}^{j}$ where $a_{i j} \in k$ and $b i+c j=\operatorname{deg} f$ 
for each term occurring in the summation. Since $x_{1}$ and $x_{2}$ do not divide $g$, we see that $f$ must contain nonzero terms of the form $a_{0 j} x_{2}^{j}$ and $a_{i 0} x_{1}^{i}$. Hence $\operatorname{deg} f$ is a multiple of $a b$, and it follows that $f$ is a polynomial in $x_{1}^{a}$ and $x_{2}^{b}$. Consequently the number of factors of $g$ in $k\left[x_{1}, x_{2}\right]$ is the number of factors of $s^{c}+t^{c}$ in $k[s, t]$ or, equivalently, the number of factors of $1+t^{c}$ in $k[t]$.

In particular, if $m_{1}$ and $m_{2}$ are relatively prime, then $g$ is irreducible and Proposition 3.1 implies that $R$ is factorial. As is well-known, $\widehat{R}$ need not be factorial; see for example, [10, Theorem III.5.2].

If $k$ is algebraically closed, then $g$ is a product of $c$ irreducible factors, and so Proposition 3.1 implies that

$$
\mathrm{Cl}(R)=(\mathbb{Z} / n \mathbb{Z})^{c-1} .
$$

Remark 3.3. The condition that the degree of $g$ is relatively prime to $n$ is certainly crucial in Proposition 3.1. In the absence of this, $\mathrm{Cl}(R)$ need not be finite, for example $\mathbb{C}\left[z, x_{1}, x_{2}, x_{3}\right] /\left(z^{3}-x_{1}^{3}-x_{2}^{3}-x_{3}^{3}\right)$ has divisor class group $\mathbb{Z}^{6}$. However, one can drop the relatively prime condition when considering hypersurfaces of the form $z^{n}-x_{0} g\left(x_{1}, \ldots, x_{d}\right)$, see also [6, Proposition 3.12]:

Corollary 3.4. Let $R=k\left[z, x_{0}, \ldots, x_{d}\right] /\left(z^{n}-x_{0} g\right)$ be a normal hypersurface over a field $k$, where $g$ is a weighted homogeneous polynomial in $x_{1}, \ldots, x_{d}$. Let $g=$ $h_{1} \cdots h_{r}$, where $h_{i} \in k\left[x_{1}, \ldots, x_{d}\right]$ are irreducible. Then

$$
\mathrm{Cl}(R)=(\mathbb{Z} / n \mathbb{Z})^{r}
$$

and the images of $\left(z, h_{1}\right), \ldots,\left(z, h_{r}\right)$ form a minimal generating set for $\mathrm{Cl}(R)$.

Proof. We may choose the degree of $x_{0}$ such that $\operatorname{deg}\left(x_{0} g\right)$ is relatively prime to $n$. The result then follows from Proposition 3.1.

We conclude with the following example.

Example 3.5. Let $k$ be a field. Corollary 3.4 implies that the divisor class group of the ring $R=k\left[x y, x^{n}, y^{n}\right]$ is $\mathbb{Z} / n \mathbb{Z}$, since $R$ is isomorphic to the hypersurface

$$
k\left[z, x_{0}, x_{1}\right] /\left(z^{n}-x_{0} x_{1}\right) .
$$

In [10, Chapter III], the divisor class group of $R$ is computed by Galois descent if $n$ is relatively prime to the characteristic of $k$, and by using derivations if $n$ equals the characteristic of $k$.

\section{References}

[1] J. Bingener and U. Storch, Zur Berechnung der Divisorenklassengruppen kompletter lokaler Ringe, Nova Acta Leopoldina (N.F.) 52 (1981), 7-63.

[2] F. Call and G. Lyubeznik, A simple proof of Grothendieck's theorem on the parafactoriality of local rings, Contemp. Math. 159 (1994), 15-18.

[3] M. Demazure, Anneaux gradués normaux, in: Introduction a la theorie des singularitiés II, pp. 35-68, Hermann, Paris, 1988.

[4] H. Flenner, Divisorenklassengruppen quasihomogener Singularitäten, J. Reine Angew. Math. 328 (1981), 128-160.

[5] A. Grothendieck, Cohomologie locale des faisceaux cohérents et théorèmes de Lefschetz locaux et globaux (SGA2), North-Holland Publishing Co., 1968.

[6] J. Lang, The divisor classes of the hypersurface $z^{p^{m}}=G\left(x_{1}, \ldots, x_{n}\right)$ in characteristic $p>0$, Trans. Amer. Math. Soc. 278 (1983), 613-634.

[7] J. Lipman, Rational singularities, with applications to algebraic surfaces and unique factorization, Inst. Hautes Etudes Sci. Publ. Math. 36 (1969), 195-279. 
[8] S. Mori, Graded factorial domains, Japan. J. Math. 3 (1977), 223-238.

[9] L. Robbiano, Factorial and almost factorial schemes in weighted projective spaces, in: Complete intersections, pp. 62-84, Lecture Notes in Math. 1092, Springer, New York, 1984.

[10] P. Samuel, Lectures on unique factorization domains, Tata Inst. Fund. Res. Stud. Math. 30, Tata Inst. Fund. Res., Bombay, 1964.

[11] G. Scheja and U. Storch, Uwe Zur Konstruktion faktorieller graduierter Integritätsbereiche, Arch. Math. (Basel) 42 (1984), 45-52.

[12] U. Storch, Fastfaktorielle Ringe, Schr. Math. Inst. Univ. Münster 36, 1967.

[13] U. Storch, Die Picard-Zahlen der Singularitäten $t_{1}^{r_{1}}+t_{2}^{r_{2}}+t_{3}^{r_{3}}+t_{4}^{r_{4}}=0$, J. Reine Angew. Math. 350 (1984), 188-202.

[14] K.-i. Watanabe, Some remarks concerning Demazure's construction of normal graded rings, Nagoya Math. J. 83 (1981), 203-211.

Department of Mathematics, University of Utah, 155 South 1400 East, Salt Lake CiтY, UT 84112, USA

E-mail address: singh@math.utah.edu

Mathematics Department, Seattle University, 901 12th Ave, Seattle, WA 98122, USA

E-mail address: spiroffs@seattleu.edu 